\title{
Processos Termodinâmicos em Linha Reta em Diagramas $p-V$
}

\author{
Straight Line Thermodynamic Processes in $p-V$ Diagrams \\ Tiago Kroetz ${ }^{* 10}$ \\ ${ }^{1}$ Universidade Tecnológica Federal do Paraná, Pato Branco, PR, Brasil
}

Recebido em 12 de Maio, 2018. Revisado em 23 de Junho, 2018. Aceito em 26 de Junho, 2018.

\begin{abstract}
Serão apresentadas propriedades gerais de processos termodinâmicos em que a pressão de um gás ideal varia linearmente com o seu volume. Denominamos esses processos termodinâmicos como "processos em linha reta". Ao se integrar a função obtida para a dependência entre a pressão e o volume foi possível escrever uma expressão geral para o trabalho associado aos processos em linha reta em termos do estado inicial do gás que sofre a transformação e das taxas de pressurização e expansão da transformação sofrida por ele. Aplicando a Primeira Lei da Termodinâmica e fazendo uso da relação entre a energia interna de um gás ideal e a sua temperatura obtivemos as expressões gerais para a variação da energia interna e para o calor absorvido por um gás ideal após passar por um processo em linha reta. O calor específico molar associado aos processos em linha reta, assim como a variação de entropia dos gases após sofrerem essas transformações, puderam ser escritos em termos das taxas de expansão e pressurização do processo. As propriedades dos processos isobáricos e isocóricos podem ser identificadas como casos especiais dos processos em linha reta e recuperadas das expressões obtidas ao se considerar as taxa de pressurização ou expansão unitárias respectivamente.
\end{abstract}

Palavras-chave: diagramas $p$ - $V$; calor específico molar; entropia; processos reversíveis.

We present here general properties of thermodynamic processes where the pressure of an ideal gas has a linear dependence with its volume. We call these thermodynamic processes as "straight-line processes". By integrating the function of gas pressure with its volume it was possible to write an expression of the work done by the gas in the straight-line processes in terms of the initial state of the gas and the rates of pressurization and expansion of the transformation. By applying the First Law of Thermodynamics and using the relation between the temperature and the internal energy of an ideal gas we obtain the general expressions of the internal energy variation and of the heat absorbed by an ideal gas after going through an straight-line process. The molar heat capacity associated with straight-line processes, as well as the entropy variation of the gases which suffer these transformations, could be written in terms of the expansion and pressurizing rates. The properties of the isobaric and isovolumetric processes can be identified as special cases of straight-line processes and recovered from the expressions obtained by considering the value of unity for the pressurizing and expansion rates respectively.

Keywords: $p$ - $V$ diagrams; molar heat capacity; entropy; reversible processes.

\section{Introdução}

Em muitas abordagens do ensino da termodinâmica, em seus diferentes níveis de profundidade, é comum a aplicação da Primeira Lei da Termodinâmica em um contexto de transformações sofridas por gases ideais. Neste sentido, o trabalho realizado por um gás é inexoravelmente apresentado como sendo a área sob a curva correspondente à variação da pressão do gás $(p)$ em função da variação de seu volume $(V)$ em processos quase-estáticos. Fazendo uso dessa associação, normalmente esquiva-se da obtenção do trabalho realizado pelos gases a partir da resolução de integrais.

Para ilustrar o cálculo do trabalho realizado por um gás, muitos exemplos e exercícios propostos nas discipli-

*Endereço de correspondência: kroetz@utfpr.edu.br nas de Física Básica recorrem a processos termodinâmicos hipotéticos em que dependência entre a pressão e o volume do gás $p(V)$ seja linear. Iremos nos referir a tais transformações como "processos em linha reta", devido à característica retilínea de sua representação em um diagrama de pressão vs. volume. Nesses casos a área sob o gráfico correspondente ao processo em um diagrama $p$ vs. $V$ pode ser obtida através da geometria de figuras planas. A partir da obtenção do trabalho realizado pelo gás, da Lei dos Gases Ideais e da Primeira Lei da Termodinâmica, também é possível obter outras propriedades do processo termodinâmico envolvido, que são: a variação de temperatura, a variação de energia interna do gás e o calor absorvido ou liberado pelo gás.

A definição do trabalho realizado por um gás como uma área em um diagrama $p$ vs. $V$ é de particular relevância no Ensino Médio, em que o Cálculo Diferencial 
e Integral não podem ser utilizados como ferramenta. Como exemplo podemos destacar alguns exercícios que exploram o cálculo da área de processos em linha reta em diagramas $p$ vs. $V$ como os indicados nas Ref. [1] e Ref. [2], sendo essas referências dois livros didáticos de nível compatível com a utilização no Ensino Médio. Mesmo no Ensino Superior em cursos de Física e Engenharias, nos períodos em que as ferramentas de Cálculo Diferencial e Integral já foram apresentadas aos discentes, as relações lineares entre $p$ e $V$ continuam presentes no ensino da termodinâmica devido ao seu valor didático. Exemplos disso são os exercícios indicados nas Ref. [3 7 . tradicionais no ensino de Física Básica em nível superior.

No entanto, podemos identificar dois problemas ao se explorar os processos em linha reta apenas a partir de valores específicos de pressão e volume iniciais e finais. O primeiro deles é o fato de não serem obtidas expressões gerais do trabalho realizado, calor absorvido, variação de energia interna e calores específicos molares envolvidos neste tipo de processo. O segundo problema é relativo ao sinal positivo ou negativo do trabalho realizado por um gás quando expandido ou contraído, respectivamente. Ao se obter o valor do trabalho através de um cálculo de área da figura plana em um diagrama $p$ vs. $V$, o sinal positivo ou negativo necessita ser atribuído a posteriori a partir da análise da variação do volume. Ao se generalizar as propriedades dos processos em linha reta, adota-se um procedimento mais sóbrio no tratamento das transformações sofridas por gases ideais. Esta abordagem, se utilizada no ensino introdutório de termodinâmica, desempenharia um papel de prelúdio para os procedimentos que se adotam em etapas subsequente do ensino, nas quais são tratadas as transformações isobáricas, isocóricas, isotérmicas e adiabáticas.

Processos termodinâmicos de expansão em linha reta de inclinação negativa receberam especial atenção em uma série de trabalhos na década de 1990 [8 10]. No primeiro trabalho 8. Dickerson e Mottmann salientaram o fato de que alguns desses processos podem ser divididos em duas etapas, sendo que durante a primeira etapa o gás absorve calor e durante a segunda etapa o gás libera calor. O estado termodinâmico intermediário em que o calor passa a diminuir é determinado pelo ponto em que a reta no diagrama $p$ vs. $V$ tangencia uma curva adiabática. Os autores investigaram as consequências dessa sutileza no contexto de um ciclo termodinâmico triangular, cuja eficiência obtida pode apresentar um valor incorreto caso seja considerado o calor total do processo em linha reta de inclinação negativa em substituição de suas duas etapas de absorção e liberação de calor. Em um trabalho subsequente [9] Valentine representou o mesmo ciclo termodinâmico utilizado no trabalho seminal de Dickerson e Mottmann em um diagrama de temperatura e entropia. Com isto, foi possível evidenciar o estado termodinâmico intermediário em que ocorre a inversão do calor através da diminuição da entropia do gás. A dependência entre a capacidade térmica e o volume ao final da expansão do mesmo processo explorado por Dickerson e Mottmann e Valentine foi investigada por Hernández em [10]. Hernandez revelou que existe uma descontinuidade entre $\infty$ e $-\infty$ no valor da capacidade térmica quando o estado final do gás tangencia uma curva isotérmica no diagrama $p$ vs. $V$, além de apresentar o valor zero quando o estado final do gás tangencia uma curva adiabática.

No presente trabalho, dedicaremo-nos a explorar as propriedades gerais de processos em linha reta em diagramas $p$ vs. $V$ considerando retas de qualquer inclinação (positiva ou negativa) e que possam representar tanto expansões como contrações do gás. Faremos isso definindo o processo em linha reta em termos de parâmetros livres que possibilitem esta diversidade de cenários. Para tanto, organizamos o trabalho de forma a apresentar na Seção 2 a expressão da dependência entre a pressão e o volume em um processo em linha reta em termos do estado inicial do gás e das taxas de expansão e pressurização. A partir da relação entre energia interna de um gás ideal e sua temperatura, apresentaremos na Seção 3 a variação da energia interna do gás em termos de seu estado inicial e das taxas de expansão e pressurização. Nas Seções 4 e 5 obteremos respectivamente as expressões gerais para o trabalho realizado por um gás e calor absorvido por ele após sofrer um processo em linha reta. O calor específico molar generalizado para qualquer processos em linha reta será explorado na Seção 6. Obteremos na Seção7] uma expressão que fornecerá a variação de entropia do gás em termos das taxas de expansão e pressurização entre seus estados posterior e anterior à tranformação sofrida pelo processo em linha reta. Ao final do trabalho, traçaremos algumas considerações finais na Seção 8

\section{Função $p(V)$}

Representamos um processo termodinâmico sofrido por um gás em um diagrama $p$ vs. $V$, conforme ilustrado na Figura 1, cuja dependência entre a pressão e volume do gás seja linear. Os pontos vermelhos representam os estados termodinâmicos inicial e final do gás. Podemos relacionar as pressões inicial $\left(p_{i}\right)$ e final $\left(p_{f}\right)$ do gás através de uma grandeza adimensional $\left(r_{p}\right)$ que denominaremos "taxa de pressurização", de maneira que $p_{f}=r_{p} p_{i}$. Também podemos relacionar os volumes inicial $\left(V_{i}\right)$ e final $\left(V_{f}\right)$ do gás através de uma grandeza adimensional $\left(r_{V}\right)$ que denominaremos "taxa de expansão", de maneira que $V_{f}=r_{V} V_{i}$.

Utilizando uma transformação linear entre as variáveis $p$ e $V$, podemos expressar a dependência $p(V)$ como

$$
p(V)=A V+B,
$$

onde $A$ e $B$ são respectivamente os coeficientes angular e linear da reta representada na Figura 1 Como $A=\frac{\Delta p}{\Delta V}$, pode-se escrever

$$
A=\frac{p_{i}\left(r_{p}-1\right)}{V_{i}\left(r_{V}-1\right)} .
$$




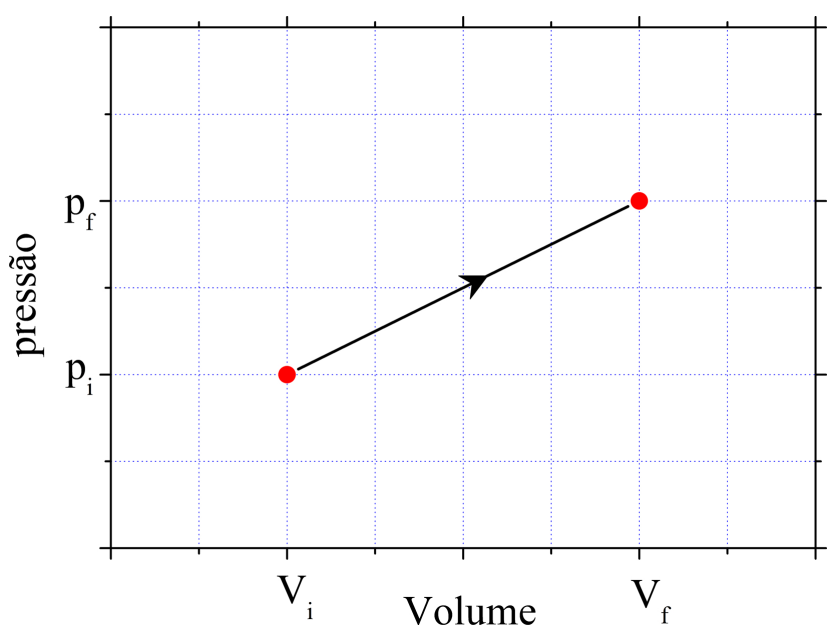

Figura 1: Processo termodinâmico de uma relação linear entre pressão e volume em um diagrama $p$ vs. $V$.

Substituindo a equação (2) em (1) para $p=p_{i}$ e $V=V_{i}$, podemos escrever o coeficiente linear da relação $p(V)$ como

$$
B=p_{i}\left[\frac{r_{V}-r_{p}}{r_{V}-1}\right]
$$

A relação expressa pela equação (1) pode representar um processo isobárico caso seja considerado $r_{p}=1$ ou um processo isocórico caso seja considerado $r_{V}=1$. Processos em linha reta em que $r_{p}<1$ consistem em despressurizações (diminuição da pressão do gás) enquanto para $r_{V}<1$ o processo consistirá em uma contração do gás (diminuição de seu volume). Um processo em que não haja variação de temperatura $(T)$ entre os estados inicial e final de um gás ideal pode ser obtido caso a relação entre as taxas de pressurização e expansão seja $r_{p} r_{V}=1$, uma vez que $p_{f} V_{f}=p_{i} V_{i}$ para gases ideais em que $T_{i}=T_{f}$. Os processos em linha reta nos quais $r_{p} r_{V}=1$ não podem ser confundidos com processos isotérmicos, uma vez que o produto $p V$ (ou a temperatura $T$ ) não se mantém constante no decorrer de todo o processo em linha reta. Um caso em que $r_{p} r_{V}=1$ corresponde a uma reta de inclinação negativa no diagrama $p$ vs. $V$ e que intercepta uma mesma curva isotérmica nos pontos associados aos estados inicial e final do processo. De maneira semelhante, um processo em linha reta em que $r_{p} r_{V}^{\gamma}=1$ leva à relação $p_{f} V_{f}^{\gamma}=p_{i} V_{i}^{\gamma}$ (onde $\gamma$ representa o coeficiente de expansão adiabática do gás) e corresponde a uma reta de inclinação negativa no diagrama $p$ vs. $V$ e que intercepta uma mesma curva adiabática nos pontos associados aos estados inicial e final do processo. Este último caso não pode ser confundido com um processo adiabático, uma vez que o gás troca calor com o meio externo no decorrer do processo em linha reta e o calor total trocado é, em geral, diferente de zero.

\section{Variação de Energia Interna}

A interpretação cinética da temperatura nos permite concluir que a energia interna $E$ de um gás ideal está relacionada com sua temperatura através da expressão

$$
E=n c_{V} T
$$

onde $n$ é o número de mols de moléculas de gás e $c_{V}$ é o calor específico molar a volume constante de um gás ideal. Em casos gerais o calor específico molar a volume constante é definido como $c_{V}=(1 / n)[\partial E(V, T) / \partial T]_{V}$ e apresenta dependência com o volume e temperatura do gás. No modelo de gás ideal adotado no presente trabalho $c_{V}$ assume valor constante e pode ser escrito como $c_{V}=\alpha R$, onde $R=8,314 J . m o l^{-1} \cdot K^{-1}$ é a constante universal dos gases ideais e $\alpha=\ell / 2$. A constante $\ell$ é um número inteiro igual ao número de graus de liberdade das moléculas. A relação entre $c_{V}$ e $\ell$ é consequência do Teorema da Equipartição de Energia [11,12]. Desta forma, para o caso de gases ideais monoatômicos temos $\ell=3$, pois os átomos possuem apenas três modos translacionais de movimento. No caso de gases ideais diatômicos temos $\ell=5$, pois as moléculas lineares possuem dois eixos de rotação ortogonais com momento de inércia considerável além dos três modos translacionais de movimento. Para gases ideais com três ou mais átomos por molécula, podemos assumir a existência de três eixos de rotação ortogonais além de três modos translacionais em cada molécula, o que corresponde a $\ell=6$. Portanto, a constante $\alpha$ assume os valores de 1,$5 ; 2,5$; ou 3 caso o gás ideal considerado seja monoatômico, diatômico ou poliatômico, respectivamente.

Por terem níveis de energia quantizados, os graus de liberdade rotacionais e vibracionais de moléculas diatômicas e poliatômicas podem não ser acessíveis para o armazenamento de energia térmica. Isso se deve à relação entre as ordens de grandeza da quantidade $k T$ (onde $k=1,381 \times 10^{-23} \mathrm{~J} / K$ é a constante de Boltzmann) e dos níveis de energia correspondentes a cada grau de liberdade das moléculas. Como os níveis de energia aproximam-se do limite do contínuo em comparação com a energia térmica à medida em que a temperatura aumenta, diz-se que nas baixas temperaturas os graus de liberdade rotacionais e vibracionais apresentam-se "congelados". Como consequência a dependência de $c_{V}$ com a temperatura pode ser relevante em gases reais diatômicos e poliatômicos em baixas temperaturas, como por exemplo o gás hidrogênio conforme pode ser observado nas ilustrações da pag. 123 da Ref. 11] e da pag. 146 da Ref. 12. No presente trabalho, por adotarmos o modelo de gases ideais, não iremos considerar a possibilidade de "congelamento de graus de liberdade" e tampouco os modos vibracionais das moléculas. Por este motivo, $c_{V}$ terá seu valor mantido constante ao longo dos processos em linha reta.

Feita essa ressalva, portanto, a equação (4) pode ser utilizada para fornecer a variação da energia interna 
de um gás ideal apenas a partir da variação de sua temperatura, através da relação

$$
\Delta E=n c_{V}\left(T_{f}-T_{i}\right) .
$$

Utilizando a Lei dos Gases Ideais, podemos escrever as temperaturas inicial $T_{i}$ e final $T_{f}$ de um gás ideal que passa por uma transformação que mantém constante o número de moléculas como sendo

$$
\begin{gathered}
T_{i}=\frac{p_{i} V_{i}}{n R}, \\
T_{f}=r_{p} r_{V} \frac{p_{i} V_{i}}{n R} .
\end{gathered}
$$

Substituindo as relações da Equações (6) e (7) na equação (5) podemos escrever a variação da energia interna de um gás ideal em termos de sua pressão e volume iniciais e das taxas de pressurização e expansão de acordo com

$$
\Delta E=\alpha p_{i} V_{i}\left(r_{p} r_{V}-1\right) .
$$

Pode-se perceber pela equação (8) que para o caso em que $T_{i}=T_{f}$ a relação entre as taxas de pressurização e expansão $r_{p} r_{V}=1$ torna nula a variação da energia interna do gás. Na Figura 2 exploramos a relação da variação da energia interna com a taxa de expansão do processo para diferentes taxas de pressurização considerando um gás ideal monoatômico $(\alpha=1,5)$.

\section{Trabalho Termodinâmico}

É possível obter uma expressão geral para o trabalho realizado por qualquer gás que passe por um processo em linha reta. Para isso fazemos uso da seguinte relação entre a função $p(V)$ e o trabalho $W$ realizado por um gás

$$
W=\int_{V_{i}}^{V_{f}} p(V) d V .
$$

Desta forma, ao integrarmos a função $p(V)$ expressa pela equação (1) e utilizando as expressões para $A$ e $B$

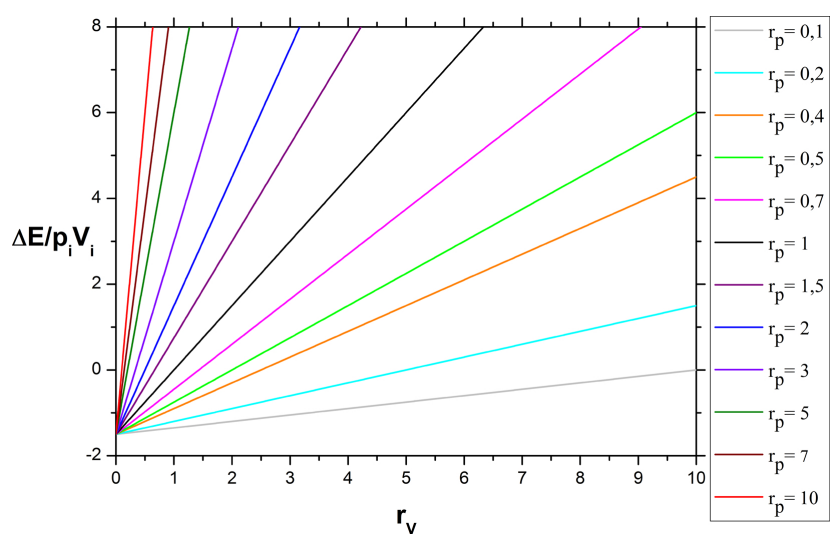

Figura 2: Variação de energia interna de um gás ideal monoatômico em função da taxa de expansão $r_{V}$ e para diferentes taxas de pressurização $r_{p}$. conforme as equações (2) e (3), escrevemos o trabalho realizado por um gás que passa por um processo em linha reta como sendo

$$
W=\frac{p_{i} V_{i}}{2}\left[\left(r_{p}+1\right)\left(r_{V}-1\right)\right] .
$$

Na Figura 3 exploramos a relação do trabalho realizado por um gás com a taxa de expansão do processo para diferentes taxas de pressurização.

A equação 10 revela que as expressões estabelecidas para o trabalho realizado em processos isocóricos e isobáricos podem ser recuperadas ao considerarmos respectivamente $r_{V}=1$ e $r_{p}=1$ na equação $(10)$. No primeiro caso obtemos o resultado $W=0$ enquanto no segundo recuperamos o resultado $W=p_{i} V_{i}\left(r_{V}-1\right)$, que também pode ser reescrito como o resultado estabelecido para processos isobáricos dos gases: $W=p_{i}\left(V_{f}-V_{i}\right)$. É possível verificar também que para contrações do gás $\left(r_{V}<1\right)$ o sinal negativo do trabalho realizado por ele é obtido a partir da equação (10), sem a necessidade de que seja inserido posteriormente como quando obtido pelo cálculo geométrico da área no diagrama $p$ vs. $V$.

\section{Calor absorvido pelo gás}

A partir das expressões gerais para a variação da energia interna de um gás ideal e para o trabalho realizado por um gás em um processo em linha reta, é possível obter uma expressão geral para o calor absorvido por um gás ideal durante este processo. Utilizando a Primeira Lei da Termodinâmica escrita na forma

$$
Q=\Delta E+W
$$

substituimos a relação de $\Delta E$ escrita na equação (8) e a relação de $W$ escrita na equação (10). Após alguma manipulação matemática, é possível expressar o calor absorvido por um gás ideal em qualquer processo em linha reta em termos do estado inicial do gás e das taxas

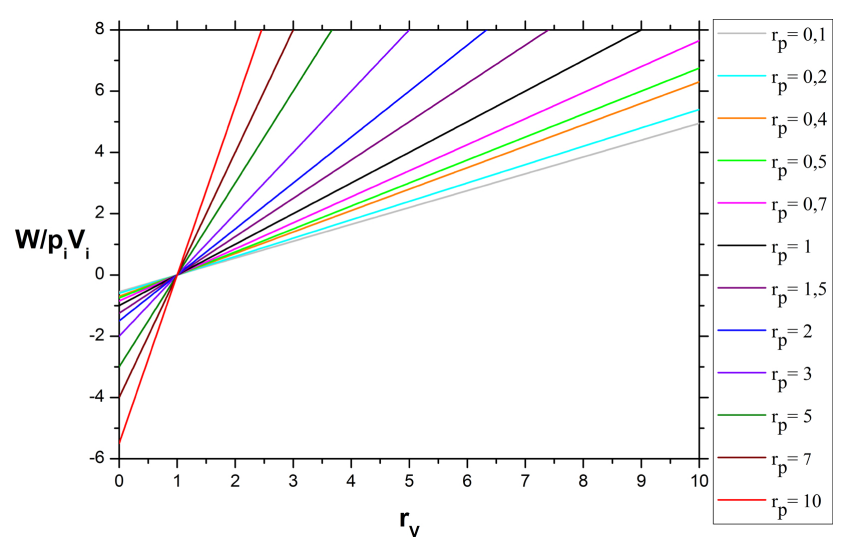

Figura 3: Trabalho realizado por um gás em um processo termodinâmico em linha reta em função da taxa de expansão $r_{V}$ e para diferentes taxas de pressurização $r_{p}$. 
de pressurização e expansão, como sendo

$$
Q=\frac{p_{i} V_{i}}{2}\left[(2 \alpha+1)\left(r_{p} r_{V}-1\right)+\left(r_{V}-r_{p}\right)\right]
$$

É possível verificar que para o caso em que $T_{i}=T_{f}$ a relação entre as taxas de expansão e pressurização $r_{p} r_{V}=1$ torna iguais os lados direitos das equações 10 e (12). Este resultado está de acordo com o esperado pela Primeira Lei da Termodinâmica, já que $\Delta E$ deve ser nulo quando não houver variação de temperatura entre os estados inicial e final e portanto $Q=W$. Além disso, quando $r_{V}=1$ a equação 12 reduz-se a $Q=$ $p_{i} V_{i} \alpha\left(r_{p}-1\right)$, que também pode ser reescrita como o resultado estabelecido para processos isocóricos de gases ideais: $Q=\alpha V_{i}\left(p_{f}-p_{i}\right)$. Já quando $r_{p}=1$ a equação 12 reduz-se a $Q=p_{i} V_{i}(\alpha+1)\left(r_{V}-1\right)$, que também pode ser reescrita como o resultado estabelecido para processos isobáricos de gases ideais: $Q=(\alpha+1) p_{i}\left(V_{f}-V_{i}\right)$.

Na Figura 4 exploramos a relação do calor absorvido por um gás ideal monoatômico $(\alpha=1,5)$ em função da taxa de expansão do processo para diferentes taxas de pressurização. Surpreendentemente, ao se considerar $\alpha=1,5$ e uma taxa de expansão de $r_{V}=0,25$ (ou seja, quando o volume do gás é reduzido para a quarta parte) o calor absorvido pelo gás em um processo em linha reta é de $Q=-1,875 p_{i} V_{i}$ independentemente da taxa de pressurização do gás. Este fato pode ser verificado tanto através da equação 12 quanto pelo cruzamento das diferentes funções correspondentes a diferentes valores de $r_{p}$ na Figura 4

\section{Calores Específicos Molares}

A capacidade térmica de um sistema termodinâmico é a constante de proporcionalidade entre o calor adicionado ao sistema e a variação de temperatura apresentada por ele. Quando o sistema em questão é um gás, é possível definir um calor específico molar " $c$ " como sendo a capacidade térmica do gás por unidade de mols de moléculas.

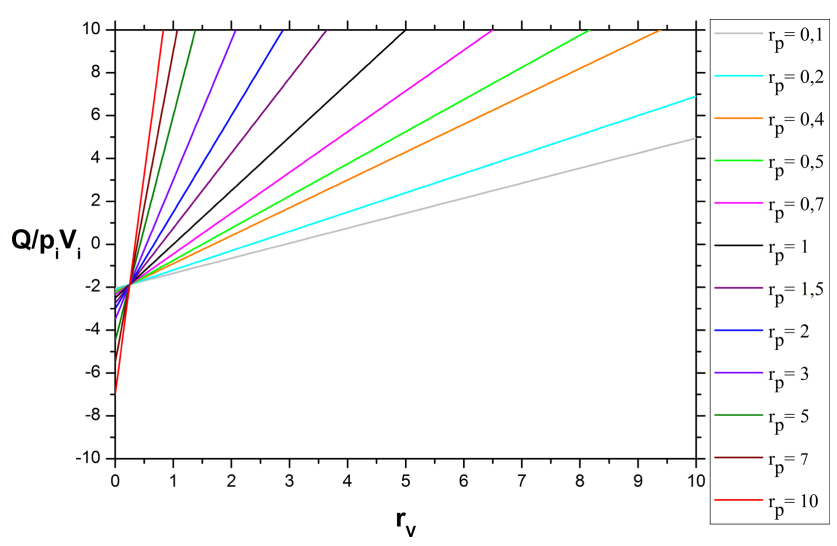

Figura 4: Calor absorvido por um gás ideal monoatômico em um processo termodinâmico em linha reta em função da taxa de expansão $r_{V}$ e para diferentes taxas de pressurização $r_{p}$.
Desta forma, ao se conhecer o calor específico molar é possível obter o calor absorvido pelo gás através de

$$
Q=n c\left(T_{f}-T_{i}\right) .
$$

Em se tratando de gases ideais, o calor específico molar é uma constante que depende apenas do processo termodinâmico envolvido e do número de átomos por moléculas do gás.

Podemos encontrar uma expressão para $c$ de diferentes processos em linha reta ao igualarmos os lados direitos das equaçãoes (12) e 13) e considerarmos as relações para $T_{i}$ e $T_{f}$ expressas pelas equações (6) e 77 , respectivamete. Com isso, ao explicitarmos $c$ encontramos a seguinte expressão:

$$
c=\frac{R}{2}\left[(2 \alpha+1)+\frac{r_{V}-r_{p}}{r_{p} r_{V}-1}\right] .
$$

A expressão do calor específico molar em processos em linha reta depende, portanto, do número de átomos por moléculas do gás e das taxas de expansão $r_{V}$ e de pressurização $r_{p}$ que definem o processo em linha reta.

A expressão para o calor específico molar a volume constante $c_{V}=\alpha R$ pode ser recuperada a partir da equação (14) ao se considerar $r_{V}=1$. De forma análoga, a expressão para calor específico molar à pressão constante $c_{p}=(\alpha+1) R$ pode ser recuperada a partir da equação (14) ao se considerar $r_{p}=1$. Utilizando a regra de L'Hôpital é possível demonstrar que $c \rightarrow c_{p}$ quando $r_{V} \rightarrow \infty$. Considerando $r_{p} r_{V}=1$ a expressão para o calor específico molar diverge, uma vez que não é possível obter o calor absorvido pelo gás a partir da variação de temperatura quando $\Delta T=0$.

Na Figura 5 exploramos a relação do calor específico molar de um gás ideal monoatômico $(\alpha=1,5)$ em função da taxa de expansão do processo em linha reta para diferentes taxas de pressurização. É possível verificar que $c / R=\alpha$ para todos os processos em que $r_{V}=1$ e que $c / R=\alpha+1$ para todos os processos em que $r_{p}=1$. Todas as funções do calor específico molar apresentam uma descontinuidade de $c \rightarrow-\infty$ para $c \rightarrow \infty$ [10 quando $r_{V} \rightarrow 1 / r_{p}$ a partir de valores à esquerda (direita) ou à direita (esquerda) respectivamente para o caso em que $r_{p}<1\left(r_{p}>1\right)$.

Destaca-se o fato de que nas vizinhanças deste limite o calor específico molar assuma valores negativos. Isso significa que uma absorção de calor provoca uma diminuição da temperatura do gás. Um exemplo específico deste caso pode ser verificado ao considerarmos $n=0,041$ mols de um gás ideal monoatômico a uma pressão inicial de $p_{i}=10^{5} \mathrm{~Pa}$ e volume inicial $V_{i}=1 L$. Ao aplicarmos a Lei dos Gases Ideais encontramos a temperatura inicial de $T_{i}=20,2^{\circ} \mathrm{C}$. Se este gás sofrer uma transformação em linha reta que o leve para uma pressão final de $p_{f}=4 \times 10^{4} \mathrm{~Pa}$ e dobre seu volume para $V_{f}=2 L$, a Lei dos Gases Ideais nos fornece uma temperatura final de $T_{f}=-38,5^{\circ} C$, resultando então em uma queda na temperatura de $\Delta T=-58,7^{\circ} \mathrm{C}$. Utilizando essa variação de 


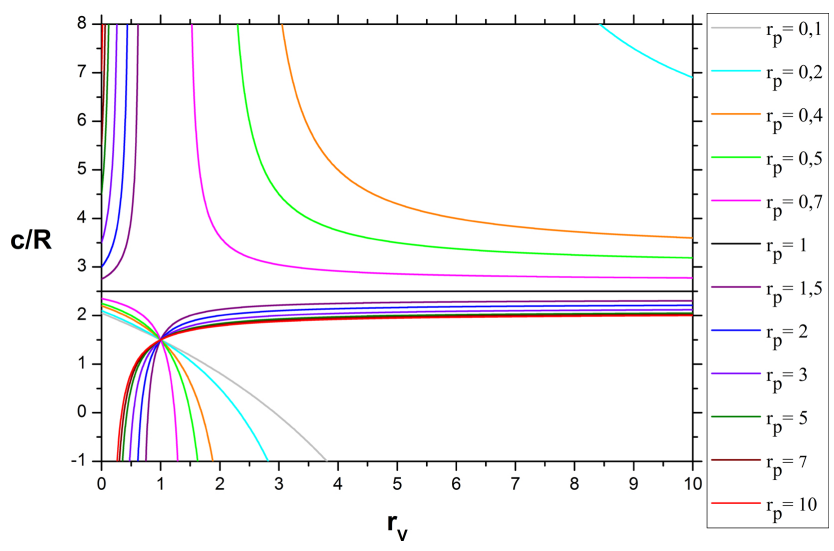

Figura 5: Calor específico molar de um gás ideal monoatômico associado a um processo termodinâmico em linha reta em função da taxa de expansão $r_{V}$ e para diferentes taxas de pressurização $r_{p}$

temperatura, a equação 5 nos fornece uma variação de energia de $\Delta E=-30 J$ enquanto ao utilizarmos $r_{V}=2$ e $r_{p}=0,4$ na equação 10 , encontraremos um trabalho realizado de $W=70 J$. Ao aplicarmos a Primeira Lei da Termodinâmica expressa pela equação 11 descobrimos que o gás precisou absorver uma quantidade de calor de $Q=40 J$ positivos. Ou seja, uma absorção de calor apesar de uma variação negativa de temperatura.

Apesar de existirem sistemas termodinâmicos capazes de apresentar calores específicos negativos 13, 14, estes sistemas normalmente exibem interações mais complexas entre seus elementos constituintes (como através de atrações gravitacionais entre partículas que constituem estrelas e galáxias, por exemplo) do que as colisões elásticas supostas entre as partículas do modelo de gás ideal. O fato de que processos termodinâmicos em linha reta sofridos por gases ideais possam resultar em calores específicos molares negativos é um indicativo, portanto, da impossibilidade de que gases reais sejam submetidos a estes processos.

\section{Variação de Entropia}

O conceito de entropia foi introduzido por Rudolf J. E. Clausius em meados do século XIX e sua interpretação foi inicialmente vinculada ao conceito de reversibilidade de processos termodinâmicos. Processos reversíveis podem ser definidos como transformações sofridas por sistemas termodinâmicos tais que, ao final da tranformação, tanto o sistema quanto a sua vizinhança podem ser restaurados aos seus estados originais sem com isso provocar qualquer alteração no resto do universo. A partir do conceito de processos reversíveis, define-se a entropia como uma variável de estado dos sistemas de forma que, ao final de um processo reversível a entropia de todo o universo permanece inalterada.

Posteriormente, as bases estatísticas da termodinâmica permitiram interpretar a entropia de um sistema como função do número de configurações microscópicas consistentes com as propriedades macroscópicas do sistema (como $p, V$ e $T$ no caso de um gás). Desta maneira, é comum relacionar entropia com a quantidade de informação adicional necessária para se especificar o estado exato de cada um dos elementos constituintes de um determinado sistema termodinâmico a partir do conhecimento de suas variáveis macroscópicas. Por este motivo, algumas interpretações da entropia a relacionam com a aleatoriedade apresentada por um sistema termodinâmico, e portanto com a "desordem" destes sistemas.

O teorema de Clausius 15] pode ser expresso matematicamente na seguinte forma

$$
\oint_{C i c} \frac{\delta Q}{T}=0,
$$

onde Cic corresponde a um caminho de integração fechado ao longo de um ciclo reversível. A equação (15) implica no fato de que o integrando $\delta Q / T$ seja uma variável de estado termodinâmico, normalmente representada por $d S$. Além disso, o resultado da integral de $d S$ ao longo de um processo reversível não-cíclico Rev pode ser escrito como $\Delta S$ e seu valor depende apenas dos estados inicial $i$ e final $f$ do processo. Denominamos a variação $\Delta S$ como a diferença de entropia entre os estados $i$ e $f$ do processo Rev, de forma que

$$
\Delta S=\int_{i}^{f} \frac{\delta Q}{T} .
$$

Como consequência do teorema de Clausius expresso pela equação 15 , o resultado de $\Delta S$ obtido pela integral de $d S$ independe do processo termodinâmico ao longo do qual se realiza a integral de caminho. Em outras palavras, qualquer outro processo termodinâmico (reversível ou irreversível) que possua os mesmos estados termodinâmicos inicial $i$ e final $f$ exibidos pelo processo reversível $R e v$, apresentará o mesmo resultado para $\Delta S$ que aquele obtido pela equação 16 . É importante destacar que a equação (16) define apenas a variação de entropia entre estados e não o valor da entropia em um determinado estado.

Embora processos irreversíveis provoquem a mesma variação de entropia no sistema que aquela provocada por processos reversíveis que compartilhem os mesmos estados termodinâmicos inicial $i$ e final $f$, a mudança de entropia nas vizinhanças do sistema não será a mesma. Definindo a variação de entropia total como a variação de entropia do sistema somada à variação de entropia da vizinhança $\Delta S_{t o t}=\Delta S_{s i s t}+\Delta S_{v i z}$, uma das possíveis formulações da Segunda Lei da Termodinâmica determina que $\Delta S_{\text {tot }}=0$ quando os processos forem reversíveis e $\Delta S_{\text {tot }}>0$ quando os processos forem irreversíveis. Na prática, processos termodinâmicos que ocorram tão lentamente a tal ponto que os estados intermediários sejam todos estados de equilíbrio com a vizinhança podem ser considerados como sendo reversíveis. Processos assim, também chamados de quase-estáticos, possuem estados 
intermediários tão bem definidos que o processo pode ser representado por uma linha contínua em um diagrama $p$ vs. $V$ 16]. Os processos em linha reta abordados no presente trabalho são todos, portanto, processos reversíveis e consequentemente $\Delta S_{\text {tot }}=0$ para todos eles.

Utilizando a forma diferencial da equação 11 podemos escrever o integrando $d S$ como

$$
\frac{\delta Q}{T}=\frac{d E}{T}+\frac{p d V}{T} .
$$

A equação (4) nos fornece a relação $d E=n c_{V} d T$ enquanto a Lei dos Gases Ideais nos fornece a relação $p / T=n R / V$. Substituindo-as em $d S$, a variação de entropia de um gás ideal pode ser obtida a partir de

$$
\Delta S=\int_{T_{i}}^{T_{f}} n c_{V} \frac{d T}{T}+\int_{V_{i}}^{V_{f}} n R \frac{d V}{V},
$$

cuja expressão após a resolução da integral definida é

$$
\Delta S=n c_{V} \ln \left(\frac{T_{f}}{T_{i}}\right)+n R \ln \left(\frac{V_{f}}{V_{i}}\right) .
$$

Substituindo as relações $c_{V}=\alpha R, T_{f} / T_{i}=r_{p} r_{V}$ e $V_{f} / V_{i}=r_{V}$ na equação (19), e após alguma manipulação matemática, podemos escrever a variação de entropia de um gás ideal em termos das taxas de expansão e pressurização do processo por ele sofrido como sendo

$$
\Delta S=n R \alpha \ln \left(r_{p} r_{V}^{\gamma}\right),
$$

onde $\gamma$ é definido como a razão entre os calores específicos $c_{p}$ e $c_{V}$, e portanto $\gamma=(\alpha+1) / \alpha$. Enfatizamos que essa relação é válida para qualquer processo em linha reta assim como para qualquer outro processo que compartilhe os mesmos estados inicial e final, uma vez que a entropia é uma variável de estado e sua variação depende apenas dos estados inicial e final do sistema submetido ao processo termodinâmico.

A expressão para a variação de entropia em processos a volume constante pode ser recuperada a partir da equação (20) ao se considerar $r_{V}=1$ de forma que $(\Delta S)_{V_{c t e}}=n c_{V} \ln \left(p_{f} / p_{i}\right)$. De forma semelhante, a variação de entropia em processos a pressão constante pode ser recuperada a partir da equação (20) ao se considerar $r_{p}=1$ de forma que $(\Delta S)_{p_{c t e}}=n c_{p} \ln \left(V_{f} / V_{i}\right)$. $\mathrm{Um}$ resultado interessante pode ser percebido ao se considerar $r_{V} r_{p}=1$, ou seja, considerando $T_{i}=T_{f}$ (mesmo que o processo não seja isotérmico). Esta situação leva à expressão para a variação de entropia escrita como $(\Delta S)_{T_{i}=T_{f}}=n R \ln \left(V_{f} / V_{i}\right)$ que é igual a $\Delta S_{T_{\text {cte }}}=Q_{T_{\text {cte }}} / T$, ou seja, a variação de entropia correspondente a um processo isotérmico. Este resultado corrobora o fato de que a variação de entropia não depende do processo termodinâmico considerado, mas apenas de seus estados inicial e final.

Por fim, pode-se notar facilmente que se $r_{p} r_{V}^{\gamma}=1$ na equação 20 teremos $\Delta S=0$. Como havíamos salientado ao final da Seção 2, um processo em linha reta em que $r_{p} r_{V}^{\gamma}=1$ corresponde a uma reta de inclinação negativa no diagrama $p$ vs. $V$ e que intercepta os pontos associados aos estados inicial e final de um processo adiabático. Por este motivo, como $(\Delta S)_{Q=0}=0$ em qualquer processo adiabático, o processo em linha reta que apresentar seus estados inicial e final coincidentes com tais processos também apresentará a mesma variação de entropia. Observamos, no entanto, que esta propriedade não garante que $Q=0 \mathrm{em}$ processos em linha reta em que $r_{p} r_{V}^{\gamma}=1$.

Na Figura 6 exploramos a relação da variação de entropia de um gás ideal monoatômico $(\alpha=1,5$ e $\gamma=1,666)$ em função da taxa de expansão de um processo termodinâmico para diferentes taxas de pressurização. Todas as funções de variação de entropia assumem o valor zero para os valores de $r_{V}$ iguais a $r_{p}^{-1 / \gamma}$. Nota-se também uma dependência crescente e monotônica da variação de entropia com ambos os parâmetros $r_{V}$ e $r_{p}$.

\section{Conclusão}

Generalizamos neste trabalho a família de processos termodinâmicos em que a pressão e o volume de um gás ideal passam por uma transformação linear, o que denominamos processos em linha reta. Os resultados aqui apresentados são de especial aplicação no ensino da termodinâmica, pois generalizam o conjunto de processos que costumeiramente são tratados em exercícios didáticos para valores específicos de pressões e volumes iniciais e finais. Mais do que isso, ilustram através de processos termodinâmicos simples a aplicação da Primeira Lei da Termodinâmica, a interpretação cinética da temperatura, o conceito de calor específico molar associado a diferentes processos e a variação de entropia em processos reversíveis. Este tratamento mais abrangente dado aos processos em linha reta assemelha-se, e portanto pode servir como uma preparação, ao tratamento utilizado em uma etapa subsequente do ensino de termodinâmica quando são abordadas as famílias de curvas isotérmi-

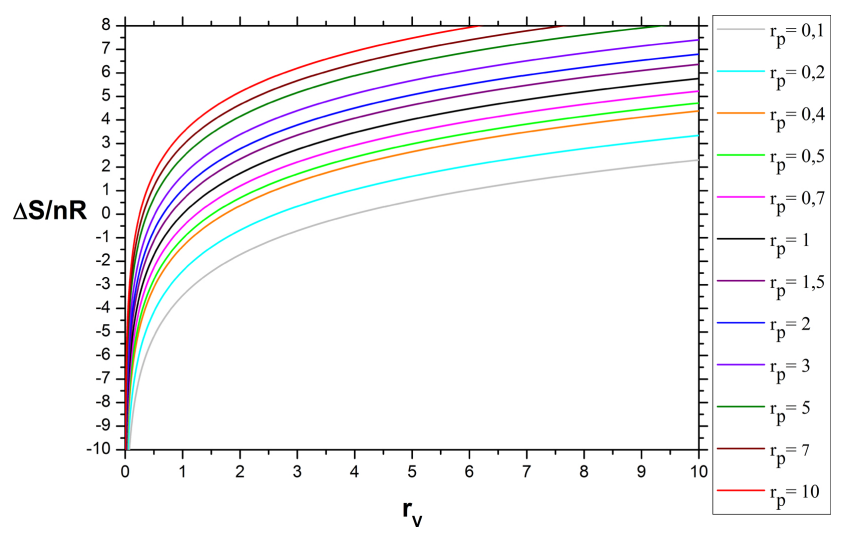

Figura 6: Variação de entropia de um gás ideal monoatômico associado a um processo termodinâmico de taxa de expansão $r_{V}$ e para diferentes taxas de pressurização $r_{p}$. 
cas e adiabáticas. Mais do que isso, as propriedades dos processos isobáricos e isocóricos, que normalmente são vistos separadamente, podem ser recuperados como casos particulares dos processos em linha reta.

\section{Referências}

[1] F. Ramalho Jr., N.G. Ferraro e P.A. Toledo Soares, Os Fundamentos da Física, V. 2 - Termologia, Óptica e Ondas (Moderna, São Paulo, 2007), 9a ed, p. 175.

[2] A. Máximo e B. Alvarenga, Física Ensino Médio, Volume 2 (Scipione, São Paulo, 2006), $1^{\mathrm{a}}$ ed, p. 150.

[3] D. Halliday, R. Resnick e J. Walker, Fundamentos de Física, Volume 2: Gravitação, Ondas e Termodinâmica (LTC, Rio de Janeiro, 2009), 8 ${ }^{\mathrm{a}}$ ed, p. 209.

[4] H.D. Young e R.A. Freedman, Sears e Zemansky Física II: Termodinâmica e Ondas (Pearson Education do Brasil, São Paulo, 2003), 10 a ed, p. 198.

[5] P.A. Tipler e G. Mosca, Física para Cientistas e Engenheiros, Volume 1: Mecânica, Oscilações e Ondas, Termodinâmica (LTC, Rio de Janeiro, 2009), $6^{\mathrm{a}}$ ed, p. 631.

[6] J.D. Cutnell e K.W. Johnson, Física, Volume 1 (LTC, Rio de Janeiro, 2016), $9^{\text {a }}$ ed, p. 441.

[7] H.M. Nussenzveig, Curso de Física Básica - vol. 2 (Blucher, São Paulo, 2002), $4^{\text {a }}$ ed, p. 187.

[8] R.H. Dickerson e J. Mottmann, Am. J. Phys. 62, 558, (1994).

[9] D.T. Valentine, Am. J. Phys. 63, 279 (1995).

[10] A.C. Hernández, Am. J. Phys. 63, 756 (1995).

[11] M.W. Zemansky, Heat and thermodynamics; an intermediate textbook (McGraw-Hill, New York, 1968), $5^{\text {a }}$ ed.

[12] R.K. Pathria, Statistical Mechanics (ButterworthHeinemann, Oxford, 1996), $2^{\mathrm{a}}$ ed.

[13] D. Lynden-Bell, Physica A. 263, 293 (1999).

[14] M. Schmidt, R. Kusche, T. Hippler, J. Donges, W. Kronmüller, B. Issendorff e H. Haberland, Phys. Rev. Lett. 86, 1191 (2001).

[15] R. Clausius, The Mechanical Theory of Heat: With Its Applications to the Steam-engine and to the Physical Properties of Bodies (J. Van Voorst, London, 1867).

[16] J.P. O'Connell e J.M. Haile, Thermodynamcs; Fundamentals for Applications (Cambridge, New York, 2005), p.22. 